\title{
Existence of positive solutions for fourth-order semipositone multi-point boundary value problems with a sign-changing nonlinear term
}

Yan Sun

Correspondence: ysun@shnu.edu. cn

Department of Mathematics, Shanghai Normal University, Shanghai 200234, People's Republic of China

\begin{abstract}
In this article, some new sufficient conditions are obtained by making use of fixed point index theory in cone and constructing some available integral operators together with approximating technique. They guarantee the existence of at least one positive solution for nonlinear fourth-order semipositone multi-point boundary value problems. The interesting point is that the nonlinear term $f$ not only involve with the first-order and the second-order derivatives explicitly, but also may be allowed to change sign and may be singular at $t=0$ and/or $t=1$. Moreover, some stronger conditions that common nonlinear term $f \geq 0$ will be modified. Finally, two examples are given to demonstrate the validity of our main results.
\end{abstract}

2000 Mathematics Subject Classification: 34B10; 34B18; 47N20.

Keywords: semipositone, positive solutions, multi-point boundary value problems

\section{Introduction}

In this article, we consider the existence of positive solutions to the following nonlinear fourth-order semipositone multi-point boundary value problems with derivatives

$$
\begin{cases}\gamma^{(4)}(t)+\lambda f\left(t, y(t), \gamma^{\prime}(t), \gamma^{\prime \prime}(t)\right)=0, & 0<t<1, \\ y(0)=\gamma^{\prime}(0)=0, \gamma^{\prime \prime}(1)=\sum_{i=1}^{m-2} \alpha_{i} y^{\prime \prime}\left(\xi_{i}\right), & \gamma^{\prime \prime \prime}(0)=\sum_{i=1}^{m-2} \beta_{i} \gamma^{\prime \prime \prime}\left(\xi_{i}\right),\end{cases}
$$

where $f \in C((0,1) \times R \times R \times R, R)$ satisfies $f\left(t, y_{1} y_{2}, y_{3}\right) \geq-p(t), p \in L^{1}((0,1),(0,+\infty)) . \lambda$ $>0, \xi_{i} \in(0,1)$ with $0<\xi_{1}<\xi_{2}<\ldots<\xi_{m-2}<1, \alpha_{i}, \beta_{i} \in[0,+\infty), i=1,2, \ldots, m-2$, are given constants satisfying $0<\sum_{i=1}^{m-2} \alpha_{i}<1,0<\sum_{i=1}^{m-2} \beta_{i}<1$. Here, by a positive solution of the problem $(1.1)$ we mean a function $y^{*}(t)$ which is positive on $(0,1)$ and satisfies the problem (1.1).

The existence of positive solutions for multi-point boundary value problems has been widely studied in recent years. For details, see [1-15] and references therein. We note that the existence of $n$ solutions and/or positive solutions to the following semipositone elastic beam equation boundary value problem

$$
\left\{\begin{array}{l}
u^{(4)}(t)=f\left(t, u(t), u^{\prime \prime}(t)\right), \quad t \in(0,1) \\
u(0)=u(1)=u^{\prime \prime}(0)=u^{\prime \prime}(1)=0
\end{array}\right.
$$

(c) 2012 Sun; licensee Springer. This is an open access article distributed under the terms of the Creative Commons Attribution License (http://creativecommons.org/licenses/by/2.0), which permits unrestricted use, distribution, and reproduction in any medium, provided the original work is properly cited. 
was obtained by Yao [13] in a Banach space setting. Gupta [3] proved the existence of positive solutions for more general multi-point boundary value problems

$$
\begin{aligned}
& x^{\prime \prime}(t)=g\left(t, x(t), x^{\prime}(t)\right)+e(t), \quad \text { a. e. } t \in(0,1) \\
& x(0)=\sum_{i=1}^{m-2} h_{i} x\left(\tau_{i}\right), \quad x^{\prime}(1)=\sum_{i=1}^{m-2} k_{i} x^{\prime}\left(\xi_{i}\right) .
\end{aligned}
$$

For further background information of multi-point boundary value problems we refer the reader to $[11,12,16]$. However, in previous work, the positivity which imposed on nonlinear term plays an important role for boundary value problems. Naturally, one is interested in establishing the existence of positive solutions for multi-point boundary value problems under the relaxed conditions.

Inspired and motivated greatly by the above mentioned works, the present work may be viewed as a direct attempt to extend the results of $[3,13]$ to a broader class of nonlinear boundary value problems in a general Banach spaces. When the nonlinearity is negative, such kinds of the problems are called semipositone problems, which occur in chemical rector theory, combustion and management of natural resources, see [11,13-16]. To our best knowledge, few results were obtained for the problem (1.1).

The purpose of the article is to establish some new criteria for the existence of positive solutions to the problem (1.1). The nonlinear term $f$ may take negative values and the nonlinearity may be sign-changing. Firstly, we employ a exchange technique and construct an integral operator for the corresponding second-order multi-point boundary value problem. Then we establish a special cone associated with concavity of functions. Finally, the existence of positive solutions for the problem (1.1) is obtained by applying fixed-point index theory. The common restriction on $f \geq 0$ is modified.

The plan of the article is as follows. Section 2 contains a number of lemmas useful to the derivation of the main results. The proof of the main results will be stated in Section 3. A class of examples are given to show that our main result is applicable to many problems in Section 4.

\section{Preliminaries and lemmas}

In this section, we shall state some necessary definitions and preliminaries.

Definition 2.1. Let $E$ be a real Banach space. A nonempty closed convex set $K \subset E$ is called a cone if it satisfies the following two conditions:

(1) $x \in K, \lambda>0$ implies $\lambda x \in K$;

(2) $x \in K,-x \in K$ implies $x=0$.

Definition 2.2. An operator $T$ is called completely continuous if it is continuous and maps bounded sets into precompact sets.

For convenience, we list the following assumptions: 
$\left(\mathbf{H}_{1}\right)$ For $i \in\{1,2, \ldots, m-2\}, \xi_{i} \in(0,1), 0<\xi_{1}<\xi_{2}<\ldots<\xi_{m-2}<1$ and $\alpha_{i}, \beta_{i} \in[0,+\infty)$ satisfying $0<\sum_{i=1}^{m-2} \alpha_{i}<1,0<\sum_{i=1}^{m-2} \beta_{i}<1$ and $0<\sum_{i=1}^{m-2} \alpha_{i} \xi_{i}<1$.

$\left(\mathbf{H}_{2}\right) f \in C((0,1) \times R \times R \times R, R)$ and there exist functions $p, q \in L^{1}((0,1),(0,+\infty))$, $g \in C(R \times R \times R,(0,+\infty))$ such that

$$
-p(t) \leq f\left(t, x_{1}, x_{2}, x_{3}\right) \leq q(t) g\left(x_{1}, x_{2}, x_{3}\right) \text { for }\left(t, x_{1}, x_{2}, x_{3}\right) \in(0,1) \times R \times R \times R .
$$

$\left(\mathbf{H}_{3}\right) \lim _{\left(\left|x_{1}\right|+\left|x_{2}\right|+\left|x_{3}\right|\right) \rightarrow+\infty} \frac{f\left(t, x_{1}, x_{2}, x_{3}\right)}{\left|x_{1}\right|+\left|x_{2}\right|+\left|x_{3}\right|}=+\infty$ for $t$ uniformly on $[0,1]$.

Remark 2.1. From $\left(\mathbf{H}_{2}\right)$ we know that for given points $t_{1}, t_{2}, \ldots, t_{m}$ on $[0,1]$, the functions $p, q=(0,1) \backslash\left\{t_{i}, i=1,2, \ldots, m\right\} \rightarrow(0,+\infty)$ are continuous and integrable, that is $0<\int_{0}^{1}(p(t)+q(t)) d t<+\infty$. The condition $\left(\mathbf{H}_{2}\right)$ also implies that $f$ may have finitely singularities at $t_{1}, t_{2}, \ldots, t_{m}$ on $[0,1]$.

Lemma 2.1. Suppose that $\left(\mathbf{H}_{1}\right)$ and $\left(\mathbf{H}_{2}\right)$ hold. Then the problem (1.1) has a positive solution if and only if the following nonlinear second-order integro-differential equation

$$
\left\{\begin{array}{l}
x^{\prime \prime}(t)+\lambda f\left(t, \int_{0}^{t}(t-u) x(u) d u, \int_{0}^{t} x(u) d u, x(t)\right)=0, \quad 0<t<1, \\
x^{\prime}(0)=\sum_{i=1}^{m-2} \beta_{i} x^{\prime}\left(\xi_{i}\right), x(1)=\sum_{i=1}^{m-2} \alpha_{i} x\left(\xi_{i}\right)
\end{array}\right.
$$

has a positive solution.

Proof. Let $y(t)$ be a positive solution of the problem (1.1) and let $x(t)=y$ " $(t)$. Then it follows from the problem (1.1) and combining with exchanging the integral sequence we know that

$$
\gamma(t)=\int_{0}^{t}(t-u) x(u) d u, \gamma^{\prime}(t)=\int_{0}^{t} x(u) d u .
$$

Thus $x(t)=y^{\prime \prime}(t)$ is a positive solution of the second-order integro-differential equation multi-point boundary value problem (2.1).

Conversely, let $x(t)$ be a positive solution of the problem (2.1), then $y(t)=\int_{0}^{t}(t-u) x(u) d u$ is a positive solution of the problem (1.1). In fact, $y^{\prime}(t)=\int_{0}^{t} x(u) d u, y^{\prime \prime}(t)=x(t)$, which implies that $y(0)=0, y^{\prime}(0)=0$. The proof is complete.

Now, let $X=C[0,1]$. Then $X$ is a real Banach space with norm $\|x\|=\max _{t \in[0,1]}|x(t)|$ for $x$ $\in C[0,1]$. Let

$$
C^{+}[0,1]=\{x \in C[0,1]: x(t) \geq 0, \quad t \in[0,1]\} .
$$

Lemma 2.2. Suppose that $\left(\mathbf{H}_{1}\right)$ holds. In addition, assume that $u(t) \in L^{1}(0,1)$ and $u$ $(t) \geq 0$. Then the following problem

$$
\left\{\begin{array}{l}
x^{\prime \prime}(t)+u(t)=0, \quad 0<t<1 \\
x^{\prime}(0)=\sum_{i=1}^{m-2} \beta_{i} x^{\prime}\left(\xi_{i}\right), x(1)=\sum_{i=1}^{m-2} \alpha_{i} x\left(\xi_{i}\right)
\end{array}\right.
$$


has a unique positive solution

$$
\begin{aligned}
& x(t)=-\int_{0}^{t}(t-s) u(s) d s+\frac{\sum_{i=1}^{m-2} \beta_{i} \int_{0}^{\xi_{i}} u(s) d s}{\sum_{i=1}^{m-2} \beta_{i}-1} t+\frac{1}{1-\sum_{i=1}^{m-2} \alpha_{i}} \int_{0}^{1}(1-s) u(s) d s \\
& -\frac{1}{1-\sum_{i=1}^{m-2} \alpha_{i}}\left(\sum_{i=1}^{m-2} \alpha_{i} \int_{0}^{\xi_{i}}\left(\xi_{i}-s\right) u(s) d s+\frac{\sum_{i=1}^{m-2} \beta_{i} \int_{0}^{\xi_{i}} u(s) d s}{\sum_{i=1}^{m-2} \beta_{i}-1}\left(1-\sum_{i=1}^{m-2} \alpha_{i} \xi_{i}\right)\right)
\end{aligned}
$$

satisfies $x(t) \geq 0, t \in[0,1]$ and

$$
\min _{t \in[0,1]} x(t) \geq w\|x\|
$$

where

$$
\omega=\frac{\sum_{i=1}^{m-2} \alpha_{i}\left(1-\xi_{i}\right)}{1-\sum_{i=1}^{m-2} \alpha_{i} \xi_{i}}
$$

Proof. From (2.2), we have $x^{\prime \prime}(t)=-u(t), 0<t<1$. For $t \in[0,1]$, integrating from 0 to $t$ we get

$$
x^{\prime}(t)=x^{\prime}(0)-\int_{0}^{t} u(s) d s .
$$

Thus

$$
x^{\prime}(0)=\sum_{i=1}^{m-2} \beta_{i} x^{\prime}\left(\xi_{i}\right)=\frac{\sum_{i=1}^{m-2} \beta_{i}}{\sum_{i=1}^{m-2} \beta_{i}-1} \int_{0}^{\xi_{i}} u(s) d s .
$$

For $t \in[0,1]$, integrating (2.6) from $t$ to 1 yields

$$
x(1)-x(t)=\frac{\sum_{i=1}^{m-2} \beta_{i}(1-t)}{\sum_{i=1}^{m-2} \beta_{i}-1} \int_{0}^{\xi_{i}} u(s) d s-\int_{0}^{t}(s-t) u(s) d s-\int_{0}^{1}(1-s) u(s) d s,
$$

which means that

$$
\begin{aligned}
-x(t)= & -\sum_{i=1}^{m-2} \alpha_{i} x\left(\xi_{i}\right)+\frac{\sum_{i=1}^{m-2} \beta_{i} \int_{0}^{\xi_{i}} u(s) d s}{\sum_{i=1}^{m-2} \beta_{i}-1}-\frac{\sum_{i=1}^{m-2} \beta_{i} \int_{0}^{\xi_{i}} u(s) d s}{\sum_{i=1}^{m-2} \beta_{i}-1} t \\
& +\int_{0}^{t}(t-s) u(s) d s-\int_{0}^{1}(1-s) u(s) d s .
\end{aligned}
$$


From (2.9), we have

$$
x\left(\xi_{i}\right)=\frac{1}{1-\sum_{i=1}^{m-2} \alpha_{i}}\left(\int_{0}^{1}(1-s) u(s) d s-\frac{\sum_{i=1}^{m-2} \beta_{i}\left(1-\xi_{i}\right) \int_{0}^{\xi_{i}} u(s) d s}{\sum_{i=1}^{m-2} \beta_{i}-1}-\int_{0}^{\xi_{i}}\left(\xi_{i}-s\right) u(s) d s\right) .
$$

It follows from (2.9) and (2.10) that

$$
\begin{aligned}
& x(t)=-\int_{0}^{t}(t-s) u(s) d s+\frac{\sum_{i=1}^{m-2} \beta_{i} \int_{0}^{\xi_{i}} u(s) d s}{\sum_{i=1}^{m-2} \beta_{i}-1} t+\int_{0}^{1}(1-s) u(s) d s \\
& +\frac{1}{1-\sum_{i=1}^{m-2} \alpha_{i}}\left(\sum_{i=1}^{m-2} \alpha_{i} \int_{0}^{1}(1-s) u(s) d s\right. \\
& \left.-\sum_{i=1}^{m-2} \alpha_{i} \int_{0}^{\xi_{i}}\left(\xi_{i}-s\right) u(s) d s-\frac{\sum_{i=1}^{m-2} \beta_{i} \int_{0}^{\xi_{i}} u(s) d s}{\sum_{i=1}^{m-2} \beta_{i}-1} \cdot\left(1-\sum_{i=1}^{m-2} \alpha_{i} \xi_{i}\right)\right) \\
& =-\int_{0}^{t}(t-s) u(s) d s+\frac{\sum_{i=1}^{m-2} \beta_{i} \int_{0}^{\xi_{i}} u(s) d s}{\sum_{i=1}^{m-2} \beta_{i}-1} t+\frac{1}{1-\sum_{i=1}^{m-2} \alpha_{i}} \int_{0}^{1}(1-s) u(s) d s \\
& -\frac{1}{1-\sum_{i=1}^{m-2} \alpha_{i}}\left(\sum_{i=1}^{m-2} \alpha_{i} \int_{0}^{\xi_{i}}\left(\xi_{i}-s\right) u(s) d s+\frac{\sum_{i=1}^{m-2} \beta_{i} \int_{0}^{\xi_{i}} u(s) d s}{\sum_{i=1}^{m-2} \beta_{i}-1}\left(1-\sum_{i=1}^{m-2} \alpha_{i} \xi_{i}\right)\right) .
\end{aligned}
$$

Combining (2.11) with $\left(\mathbf{H}_{1}\right)$ we know that

$$
\begin{aligned}
x(0)= & \frac{1}{1-\sum_{i=1}^{m-2} \alpha_{i}}\left(\int_{0}^{1}(1-s) u(s) d s-\sum_{i=1}^{m-2} \alpha_{i} \int_{0}^{\xi_{i}}\left(\xi_{i}-s\right) u(s) d s\right. \\
& \left.-\frac{\sum_{i=1}^{m-2} \beta_{i} \int_{0}^{\xi_{i}} u(s) d s}{\sum_{i=1}^{m-2} \beta_{i}-1}\left(1-\sum_{i=1}^{m-2} \alpha_{i} \xi_{i}\right)\right) \\
\geq & \frac{1}{1-\sum_{i=1}^{m-2} \alpha_{i}}\left(\sum_{i=1}^{m-2} \alpha_{i} \int_{0}^{1}(1-s) u(s) d s-\sum_{i=1}^{m-2} \alpha_{i} \int_{0}^{\xi_{i}}\left(\xi_{i}-s\right) u(s) d s\right. \\
& \left.-\frac{\sum_{i=1}^{m-2} \beta_{i} \int_{0}^{\xi_{i}} u(s) d s}{\sum_{i=1}^{m-2} \beta_{i}-1}\left(1-\sum_{i=1}^{m-2} \alpha_{i} \xi_{i}\right)\right) \\
\geq & \frac{\sum_{i=1}^{m-2} \alpha_{i}}{1-\sum_{i=1}^{m-2} \alpha_{i}} \int_{\xi_{i}}^{1}(1-s) u(s) d s+\frac{\sum_{i=1}^{m-2} \beta_{i} \int_{0}^{\xi_{i}} u(s) d s\left(1-\sum_{i=1}^{m-2} \alpha_{i} \xi_{i}\right)}{\left(1-\sum_{i=1}^{m-2} \beta_{i}\right)\left(1-\sum_{i=1}^{m-2} \alpha_{i}\right)} \geq 0 .
\end{aligned}
$$

From the fact that $x^{\prime \prime}(t)=-u(t) \leq 0$, we know that the graph of $x(t)$ is concave on $[0,1]$. 
Thus

If $x(1) \geq 0$, we know that $x(t) \geq 0$ for all $t \in[0,1]$.

If $x(1)<0$, from the concavity of $x$ once again we know that

$$
\frac{x\left(\xi_{i}\right)}{\xi_{i}} \geq \frac{x(1)}{1}
$$

for $i \in\{1,2, \ldots, m-2\}$. This implies

$$
x(1)=\sum_{i=1}^{m-2} \alpha_{i} x\left(\xi_{i}\right) \geq \sum_{i=1}^{m-2} \alpha_{i} \xi_{i} x(1),
$$

which contracts with the fact $0<\sum_{i=1}^{m-2} \alpha_{i} \xi_{i}<1$. Thus we know that (2.4) holds.

Again from $x^{\prime \prime}(t)=-u(t) \leq 0$, we see that $x^{\prime}(t)$ is non-increasing on $(0,1)$. Combining the condition $0<\sum_{i=1}^{m-2} \beta_{i}<1$ we have $x^{\prime}(0) \leq 0$ and $x^{\prime}(t)=x^{\prime}(0)-\int_{0}^{t} u(s) d s \leq 0$ for $t \in(0$, $1)$. Hence $x(t)$ is non-increasing on $(0,1)$. By making use of the concavity of $x(t)$ on $(0,1)$ we get $\|x\|=x(0)$ and $\min _{t \in[0,1]} x(t)=x(1)$. Therefore, for all $i=1,2, \ldots, m-2$, we obtain

$$
\begin{aligned}
\|x\| & =x(0) \leq x(1)+\frac{x\left(\xi_{i}\right)-x(1)}{1-\xi_{i}}(1-0)=x(1)+\frac{x\left(\xi_{i}\right)}{1-\xi_{i}}-\frac{x(1)}{1-\xi_{i}} \\
& =\frac{x\left(\xi_{i}\right)-x(1) \xi_{i}}{1-\xi_{i}}=\frac{1-\sum_{i=1}^{m-2} \alpha_{i} \xi_{i}}{\sum_{i=1}^{m-2} \alpha_{i}\left(1-\xi_{i}\right)} x(1),
\end{aligned}
$$

which implies that

$$
\min _{t \in[0,1]} x(t) \geq \frac{\sum_{i=1}^{m-2} \alpha_{i}\left(1-\xi_{i}\right)}{1-\sum_{i=1}^{m-2} \alpha_{i} \xi_{i}}\|x\|=\omega\|x\|
$$

where $\omega$ is given by (2.5). This completes the proof.

Lemma 2.3. Suppose that $\left(\mathbf{H}_{1}\right)$ holds. In addition, assume that $p \in L^{1}((0,1),(0,+\infty))$. Then the following boundary value problem

$$
\left\{\begin{array}{l}
x^{\prime \prime}(t)+\lambda p(t)=0, \quad 0<t<1 \\
x^{\prime}(0)=\sum_{i=1}^{m-2} \beta_{i} x^{\prime}\left(\xi_{i}\right), x(1)=\sum_{i=1}^{m-2} \alpha_{i} x\left(\xi_{i}\right)
\end{array}\right.
$$

has a unique positive solution $z$ satisfying $z(t) \geq 0, t \in[0,1], \min _{t \in[0,1]} z(t) \geq \omega\|z\|_{\text {and }}$

$$
z(t) \leq \lambda G \omega \int_{0}^{1} p(s) d s,
$$

where

$$
G=\left(\frac{1}{1-\sum_{i=1}^{m-2} \alpha_{i}}+\frac{\sum_{i=1}^{m-2} \beta_{i}\left(1-\sum_{i=1}^{m-2} \alpha_{i} \xi_{i}\right)}{\left(1-\sum_{i=1}^{m-2} \alpha_{i}\right)\left(1-\sum_{i=1}^{m-2} \beta_{i}\right)}\right) \omega^{-1},
$$


$\omega$ is given by (2.5).

Proof. From Lemma 2.2. we have $z(t) \geq 0$ and $\min _{t \in[0,1]} z(t) \geq \omega\|z\|_{t} t \in[0,1]$. By making use of (2.3) we get

$$
\begin{aligned}
z(t) & \leq \frac{\lambda}{1-\sum_{i=1}^{m-2} \alpha_{i}}\left(\int_{0}^{1}(1-s) p(s) d s-\sum_{i=1}^{m-2} \alpha_{i} \int_{0}^{\xi_{i}}\left(\xi_{i}-s\right) p(s) d s\right. \\
& \left.+\frac{\sum_{i=1}^{m-2} \beta_{i} \int_{0}^{\xi_{i}} p(s) d s}{1-\sum_{i=1}^{m-2} \beta_{i}}\right) \cdot\left(1-\sum_{i=1}^{m-2} \alpha_{i} \xi_{i}\right) \\
& \leq \frac{1-\sum_{i=1}^{m-2} \alpha_{i}}{\left.\int_{0}^{1} p(s) d s+\frac{\sum_{i=1}^{m-2} \beta_{i} \int_{0}^{1} p(s) d s}{1-\sum_{i=1}^{m-2} \beta_{i}}\left(1-\sum_{i=1}^{m-2} \alpha_{i} \xi_{i}\right)\right)} \\
& =\lambda \int_{0}^{1} p(s) d s\left(\frac{1}{1-\sum_{i=1}^{m-2} \alpha_{i}}+\frac{\sum_{i=1}^{m-2} \beta_{i}\left(1-\sum_{i=1}^{m-2} \alpha_{i} \xi_{i}\right)}{\left(1-\sum_{i=1}^{m-2} \alpha_{i}\right)\left(1-\sum_{i=1}^{m-2} \beta_{i}\right)}\right)=\lambda G \omega \int_{0}^{1} p(s) d s .
\end{aligned}
$$

The proof is complete. $\square$

Let

$$
[v(t)]^{*}=\left\{\begin{array}{l}
v(t), v(t) \geq 0, \quad 0<t<1 \\
0, \quad v(t)<0, \quad 0 .
\end{array}\right.
$$

and

$$
\begin{gathered}
F\left(t,[x(t)-z(t)]^{*}\right)=f\left(t, \int_{0}^{t}(t-u)[x(u)-z(u)]^{*} d u, \int_{0}^{t}[x(u)-z(u)]^{*} d u,\right. \\
\left.[x(t)-z(t)]^{*}\right)+p(t) .
\end{gathered}
$$

Lemma 2.4. Suppose that $\left(\mathbf{H}_{1}\right)$ and $\left(\mathbf{H}_{2}\right)$ hold. Then the following nonlinear secondorder integro-differential equation boundary value problem

$$
\left\{\begin{aligned}
x^{\prime \prime}(t)+\lambda\left[f \left(t, \int_{0}^{t}(t-u)[x(u)-z(u)]^{*} d u, \int_{0}^{t}[x(u)-z(u)]^{*} d u,\right.\right. \\
{\left.\left.[x(t)-z(t)]^{*}\right)+p(t)\right]=0, \quad 0<t<1, } \\
x^{\prime}(0)=\sum_{i=1}^{m-2} \beta_{i} x^{\prime}\left(\xi_{i}\right), x(1)=\sum_{i=1}^{m-2} \alpha_{i} x\left(\xi_{i}\right)
\end{aligned}\right.
$$

has a positive solution $x(t)$ with $x(t) \geq z(t)$ for $t \in[0,1]$ if and only if $y(t)=x(t)-z(t)$ is a nonnegative solution (positive on $(0,1)$ ) of the problem (2.1).

Proof. Assume that $y(t)=x(t)-z(t)$ is a nonnegative solution (positive on $(0,1)$ ) of the problem (2.1). Then we know that $x(t) \geq z(t)$ and

$$
\gamma^{\prime \prime}(t)+\lambda f\left(t, \int_{0}^{t}(t-u) y(u) d u, \int_{0}^{t} \gamma(u) d u, y(t)\right)=0 .
$$


Noticing that $z$ is a positive solution of the problem (2.13). Thus we get

$$
-x^{\prime \prime}(t)=\lambda\left[f\left(t, \int_{0}^{t}(t-u)[x(u)-z(u)]^{*} d u, \int_{0}^{t}[x(u)-z(u)]^{*} d u,[x(t)-z(t)]^{*}\right)+p(t)\right]
$$

Therefore $x(t)$ is a positive solution of the problem (2.15) with $x(t) \geq z(t)$ for $t \in[0,1]$.

Conversely, we assume that $x(t)$ and $z(t)$ are positive solutions of the problem (2.15) and the problem (2.13), respectively, and it implies that the boundary conditions of the problem (2.13) are also satisfied. Thus $y(t)=x(t)-z(t)$ is a nonnegative solution (positive on $(0,1))$ of the problem $(2.1)$. The proof is complete.

Remark 2.2. Combining Lemma 2.4. with Lemma 2.1. we know that if the problem (2.15) has a positive solution, then the fourth-order multi-point boundary value problem (1.1) has a positive solution. So, we need only to study the problem (2.15).

Remark 2.3. For any fixed $x \in C^{+}[0,1]$, let $L=\max _{t \in[0,1]} x(t)$. Noticing that $[x(u)-z(u)]^{*}$ $\leq x(u) \leq L$ and $\left|\int_{0}^{s}(s-u)[x(u)-z(u)]^{*} d u\right| \leq \int_{0}^{1} L d u=L$, by virtue of $\left(\mathbf{H}_{2}\right)$, we obtain

$$
\begin{aligned}
\int_{0}^{1} F\left(s,[x(s)-z(s)]^{*}\right) d s \leq & \int_{0}^{1}\left[q ( s ) g \left(\int_{0}^{s}(s-u)[x(u)-z(u)]^{*} d u,\right.\right. \\
& \left.\left.\int_{0}^{s}[x(u)-z(u)]^{*} d u,[x(s)-z(s)]^{*}\right)+p(s)\right] d s \\
& \leq \int_{0}^{1}(p(s)+M q(s)) d s \\
& \leq M \int_{0}^{1}(p(s)+q(s)) d s<+\infty,
\end{aligned}
$$

where

$$
M=\max _{\left(\left|u_{1}\right|,\left|u_{2}\right|,\left|u_{3}\right|\right) \in[0, L] \times[0, L] \times[0, L]} g\left(\left|u_{1}\right|,\left|u_{2}\right|,\left|u_{3}\right|\right)+1 .
$$

We introduce an integral mapping $T: C^{+}[0,1] \rightarrow C^{+}[0,1]$ defined by

$$
\begin{aligned}
(T x)(t) & =-\lambda\left(\int_{0}^{t}(t-s) F\left(s,[x(s)-z(s)]^{*}\right) d s\right. \\
& \left.-\frac{t \sum_{i=1}^{m-2} \beta_{i} \int_{0}^{\xi_{i}} F\left(s,[x(s)-z(s)]^{*}\right) d s}{1-\sum_{i=1}^{m-2} \beta_{i}}\right) \\
& +\frac{\lambda}{1-\sum_{i=1}^{m-2} \alpha_{i}}\left(\int_{0}^{1}(1-s) F\left(s,[x(s)-z(s)]^{*}\right) d s\right. \\
& -\sum_{i=1}^{m-2} \alpha_{i} \int_{0}^{\xi_{i}}\left(\xi_{i}-s\right) F\left(s,[x(s)-z(s)]^{*}\right) d s \\
& \left.+\frac{\sum_{i=1}^{m-2} \beta_{i} \int_{0}^{\xi_{i}} F\left(s,[x(s)-z(s)]^{*}\right) d s}{1-\sum_{i=1}^{m-2} \beta_{i}}\left(1-\sum_{i=1}^{m-2} \alpha_{i} \xi_{i}\right)\right) .
\end{aligned}
$$


Denote

$$
K=\left\{y \in C^{+}[0,1]: \min _{0 \leq t \leq 1} \gamma(t) \geq \omega\|y\|\right\},
$$

where $\omega$ is given by the problem (2.5). It is obvious that $K$ is a positive cone of $C$ $[0,1]$.

Lemma 2.5. Suppose that $\left(\mathbf{H}_{1}\right)-\left(\mathbf{H}_{3}\right)$ hold. Then $T: K \rightarrow K$ is a completely continuous operator.

Proof. It follows from Lemma 2.2. we see $T(K) \subset K$. Combining $\left(\mathbf{H}_{1}\right)$ with (2.18) we know that $T(K)$ is equicontinuous and uniformly bounded. In fact, let $D \subset C^{+}[0,1]$ be a bounded set. Then there exists $M_{0}>0$ such that $\|x\| \leq M_{0}$ for all $x \in D$. By virtue of $\left(\mathbf{H}_{2}\right)$ we obtain

$$
\begin{aligned}
& |(T x)(t)|=\mid-\lambda \int_{0}^{t}(t-s) F\left(s,[x(s)-z(s)]^{*}\right) d s-\frac{\lambda \sum_{i=1}^{m-2} \beta_{i} \int_{0}^{\xi_{i}} F\left(s,[x(s)-z(s)]^{*}\right) d s}{1-\sum_{i=1}^{m-2} \beta_{i}} t \\
& +\frac{\lambda}{1-\sum_{i=1}^{m-2} \alpha_{i}}\left(\int_{0}^{1}(1-s) F\left(s,[x(s)-z(s)]^{*}\right) d s-\sum_{i=1}^{m-2} \alpha_{i} \int_{0}^{\xi_{i}}\left(\xi_{i}-s\right) F\left(s,[x(s)-z(s)]^{*}\right) d s\right. \\
& \left.-\frac{\sum_{i=1}^{m-2} \beta_{i} \int_{0}^{\xi_{i}} F\left(s,[x(s)-z(s)]^{*}\right) d s}{\sum_{i=1}^{m-2} \beta_{i}-1}\left(1-\sum_{i=1}^{m-2} \alpha_{i} \xi_{i}\right)\right) \mid \\
& \leq \mid \frac{\lambda}{1-\sum_{i=1}^{m-2} \alpha_{i}}\left(\int_{0}^{1} F\left(s,[x(s)-z(s)]^{*}\right) d s\right. \\
& \left.+\frac{\sum_{i=1}^{m-2} \beta_{i}\left(1-\sum_{i=1}^{m-2} \alpha_{i} \xi_{i}\right)}{1-\sum_{i=1}^{m-2} \beta_{i}} \int_{0}^{1} F\left(s,[x(s)-z(s)]^{*}\right) d s\right) \mid \\
& =\frac{\lambda}{1-\sum_{i=1}^{m-2} \alpha_{i}}\left(1+\frac{\sum_{i=1}^{m-2} \beta_{i}\left(1-\sum_{i=1}^{m-2} \alpha_{i} \xi_{i}\right)}{1-\sum_{i=1}^{m-2} \beta_{i}}\right) \int_{0}^{1} F\left(s,[x(s)-z(s)]^{*}\right) d s \\
& \leq \frac{\lambda M\left(1-\sum_{i=1}^{m-2} \beta_{i} \sum_{i=1}^{m-2} \alpha_{i} \xi_{i}\right)}{\left(1-\sum_{i=1}^{m-2} \alpha_{i}\right)\left(1-\sum_{i=1}^{m-2} \beta_{i}\right)} \int_{0}^{1}(q(s)+p(s)) d s<+\infty,
\end{aligned}
$$

which implies that $T(K)$ is uniformly bounded.

On the other hand, for all $x \in D$, once again from $\left(\mathbf{H}_{2}\right)$ we have

$$
\begin{aligned}
0 \leq\left|(T x)^{\prime}(t)\right| & =\lambda\left|-\int_{0}^{t} F\left(s,[x(s)-z(s)]^{*}\right) d s-\frac{\sum_{i=1}^{m-2} \beta_{i} \int_{0}^{\xi_{i}} F\left(s,[x(s)-z(s)]^{*}\right) d s}{1-\sum_{i=1}^{m-2} \beta_{i}}\right| \\
& \leq \lambda\left|\int_{0}^{1} F\left(s,[x(s)-z(s)]^{*}\right) d s+\frac{\sum_{i=1}^{m-2} \beta_{i}}{1-\sum_{i=1}^{m-2} \beta_{i}} \int_{0}^{1} F\left(s,[x(s)-z(s)]^{*}\right) d s\right| \\
& =\frac{\lambda}{1-\sum_{i=1}^{m-2} \beta_{i}} \int_{0}^{1} F\left(s,[x(s)-z(s)]^{*}\right) d s \\
& \leq \frac{\lambda M}{1-\sum_{i=1}^{m-2} \beta_{i}} \int_{0}^{1}[q(s)+p(s)] d s=\frac{\lambda M M^{*}}{1-\sum_{i=1}^{m-2} \beta_{i}}<+\infty,
\end{aligned}
$$


here $M^{*}=\int_{0}^{1}[p(s)+q(s)] d s$. So, for any $0 \leq t_{1}<t_{2} \leq 1$, and for all $x \in D$, we get

$$
\left|(T x)\left(t_{1}\right)-(T x)\left(t_{2}\right)\right|=\left|\int_{t_{1}}^{t_{2}}(T x)^{\prime}(t) d t\right| \leq \frac{\lambda M M^{*}}{1-\sum_{i=1}^{m-2} \beta_{i}}\left|t_{2}-t_{1}\right| .
$$

By the absolutely continuous of integral, we know that $T(K)$ is equicontinuous on $[0,1]$. Thus, an application of the Ascoli-Arzela theorem we know that $T(K)$ is a relatively compact set.

Now we show that $T$ is continuous. Let $x_{n} \rightarrow x^{*}(n \rightarrow \infty), x_{n}, x^{* \prime} \in C^{+}[0,1]$. It follows from the Lebesgue control convergence theorem that we obtain

$$
\left\|\left(T x_{n}\right)(t)-\left(T x^{*}\right)(t)\right\| \rightarrow 0(n \rightarrow \infty), t \in[0,1] .
$$

Therefore $T: K \rightarrow K$ is a completely continuous operator. The proof is complete. $\square$

Lemma 2.6. [17] Let $X=(X,\|\cdot\|)$ be a Banach space and $K \subset X$ be a cone. For $r>0$ define $K_{r}=\{u \in K:\|u\|<r\}$. Assume that $T: \bar{K}_{r} \rightarrow K$ is a completely continuous operator, such that $T u \neq u$ for $u \in \partial K_{r}=\{u \in K:\|u\|=r\}$, and

(1) If $\|T u\| \geq\|u\|$ for $u \in \partial K_{r}$, then $i\left(T, K_{r}, K\right)=0$,

(2) If $\|T u\| \leq\|u\|$ for $u \in \partial K_{r}$, then $i\left(T, K_{r}, K\right)=1$.

\section{Main results}

In this section, we shall apply Lemma 2.6. to establish the existence of at least one positive solutions of the problem (1.1).

Theorem 3.1. Suppose that $\left(\mathbf{H}_{1}\right)-\left(\mathbf{H}_{3}\right)$ hold. Then there exists sufficiently small $\lambda^{*}>0$ such that the problem (1.1) has at least one positive solution for any $\lambda \in\left(0, \lambda^{*}\right)$.

Proof. Let $r>1$ and $\lambda \in\left(0, \lambda^{*}\right)$ be fixed, where

$$
\begin{aligned}
& \lambda^{*}=\min \left\{r\left[\frac{M_{1} \int_{0}^{1}(p(s)+q(s)) d s\left(1-\sum_{i=1}^{m-2} \beta_{i} \sum_{i=1}^{m-2} \alpha_{i} \xi_{i}\right)}{\left(1-\sum_{i=1}^{m-2} \alpha_{i}\right)\left(1-\sum_{i=1}^{m-2} \beta_{i}\right)}\right]^{-1}, \frac{r}{G \int_{0}^{1} p(s) d s}, 1\right\} \\
& \text { here } M_{1}=\max _{\left(\left|u_{1}\right|,\left|u_{2}\right|,\left|u_{3}\right|\right) \in[0, r] \times[0, r] \times[0, r]}\left\{g\left(\left|u_{1}\right|,\left|u_{2}\right|,\left|u_{3}\right|\right)\right\}+1 \text {. Choose } \Omega_{r}=\left\{x \in C^{+}[0,1]\right. \text { : }
\end{aligned}
$$
$\|x\|<r\}$. If there is a fixed point on $\partial \Omega_{r}$, we complete the proof. Without loss of generality, we may assume that there is no fixed point on $\partial \Omega_{r}$. Thus, for any $x \in K \cap \partial \Omega_{r}$, from (3.1) we get

$$
\begin{aligned}
(T x)(t) \leq & \frac{\lambda}{1-\sum_{i=1}^{m-2} \alpha_{i}} \int_{0}^{1}(1-s) F\left(s,[x(s)-z(s)]^{*}\right) d s \\
& +\frac{\lambda \sum_{i=1}^{m-2} \beta_{i} \int_{0}^{\xi_{i}} F\left(s,[x(s)-z(s)]^{*}\right) d s}{\left(1-\sum_{i=1}^{m-2} \alpha_{i}\right)\left(1-\sum_{i=1}^{m-2} \beta_{i}\right)}\left(1-\sum_{i=1}^{m-2} \alpha_{i} \xi_{i}\right) \\
& \leq \frac{\lambda M_{1}\left(1-\sum_{i=1}^{m-2} \beta_{i} \sum_{i=1}^{m-2} \alpha_{i} \xi_{i}\right) \int_{0}^{1}(p(s)+q(s)) d s}{\left(1-\sum_{i=1}^{m-2} \alpha_{i}\right)\left(1-\sum_{i=1}^{m-2} \beta_{i}\right)}<r=\|x\| .
\end{aligned}
$$


So

$\|T x\|<\|x\|$ for all $x \in K \cap \partial \Omega_{r}$.

It follows from Lemma 2.6. we know that

$$
i\left(T, \Omega_{r}, \Omega\right)=1 \text {. }
$$

Let $d$ be a real number such that

$$
\frac{1}{2} \lambda d w \geq r .
$$

Choose $R>\max \{r, \lambda d \omega+1\}$ such that if $\varphi>\frac{1}{r} R \omega$, then

$$
\frac{F(t, \varphi)}{\varphi} \geq d \text { for } t \in[0,1]
$$

and

$$
1-\frac{\lambda G p(s)}{R} \int_{0}^{1} p(s) d s \geq \frac{1}{r} .
$$

Let $z=p(s) \tilde{z}$, where $\tilde{z}$ is the unique solution of the problem (2.13). Denote

$$
\Omega_{R}=\left\{x \in C^{+}[0,1]:\|x\|<R\right\} .
$$

From (2.14) we have

$$
\begin{aligned}
z(s) & =p(s) \tilde{z}(s) \leq \lambda p(s) G w \int_{0}^{1} p(s) d s \\
& \leq \lambda p(s) G \int_{0}^{1} p(s) d s \frac{x(s)}{\|x\|}, \forall x \in K \cap \partial \Omega_{R} .
\end{aligned}
$$

Thus

$$
x(s)-z(s) \geq\left(1-\frac{\lambda G p(s) \int_{0}^{1} p(s) d s}{R}\right) x(s) .
$$

Combining (3.7) with (3.5) and by making use of Lemma 2.2. we know that

$$
x(s)-z(s) \geq \frac{1}{r} x(s) \geq \frac{1}{r} \min _{s \in[0,1]} x(s) \geq \frac{1}{r}\|x\| w \geq \frac{1}{r} R w, s \in[0,1], x \in K \cap \partial \Omega_{R} .
$$

From (3.8) together with (3.4), we see that

$$
F(s,(x-z)) \geq d(x-z) \geq \frac{R d w}{r}, s \in[0,1] .
$$


Then

$$
\begin{aligned}
& \int_{0}^{s}(s-u)[x(u)-z(u)]^{*} d u+\int_{0}^{s}[x(u)-z(u)]^{*} d u+[x(s)-z(s)]^{*} \\
& =\int_{0}^{s}(s-u)[x(u)-z(u)] d u+\int_{0}^{s}[x(u)-z(u)] d u+[x(s)-z(s)] \\
& >[x(s)-z(s)] \geq \frac{1}{r} R w, s \in[0,1] .
\end{aligned}
$$

Thus, for any $x \in K \cap \partial \Omega_{R}$, it follows from $\left(\mathbf{H}_{3}\right)$ we know that

$$
\begin{aligned}
& f\left(s, \int_{0}^{s}(s-u)[x(u)-z(u)]^{*} d u, \int_{0}^{s}[x(u)-z(u)]^{*} d u,[x(s)-z(s)]^{*}\right) \\
& \geq d\left(\int_{0}^{s}(s-u)[x(u)-z(u)]^{*} d u+\int_{0}^{s}[x(u)-z(u)]^{*} d u+[x(s)-z(s)]^{*}\right)+p(s) \\
& \geq d[x(s)-z(s)]^{*} \geq d[x(s)-z(s)] \geq \frac{d R w}{r}, s \in[0,1] .
\end{aligned}
$$

Therefore, in view of (2.18) and (3.9) together with (3.3) we get

$$
\begin{aligned}
& (T x)(0)=\frac{\lambda}{1-\sum_{i=1}^{m-2} \alpha_{i}}\left(\int_{0}^{1}(1-s) F\left(s,[x(s)-z(s)]^{*}\right) d s\right. \\
& -\sum_{i=1}^{m-2} \alpha_{i} \int_{0}^{\xi_{i}}\left(\xi_{i}-s\right) F\left(s,[x(s)-z(s)]^{*}\right) d s \\
& \left.-\frac{\sum_{i=1}^{m-2} \beta_{i} \int_{0}^{\xi_{i}} F\left(s,[x(s)-z(s)]^{*}\right) d s}{\sum_{i=1}^{m-2} \beta_{i}-1}\left(1-\sum_{i=1}^{m-2} \alpha_{i} \xi_{i}\right)\right) \\
& \geq \frac{\lambda}{1-\sum_{i=1}^{m-2} \alpha_{i}}\left[\int_{0}^{1}(1-s) F\left(s,[x(s)-z(s)]^{*}\right) d s\right. \\
& \left.-\sum_{i=1}^{m-2} \alpha_{i} \int_{0}^{1}\left(\xi_{i}-s\right) F\left(s,[x(s)-z(s)]^{*}\right) d s\right] \\
& =\lambda \int_{0}^{1}(1-s) F\left(s,[x(s)-z(s)]^{*}\right) d s \\
& =\lambda \int_{0}^{1}(1-s)\left[f \left(s, \int_{0}^{s}(s-u)[x(u)-z(u)]^{*} d u,\right.\right. \\
& \left.\left.\int_{0}^{s}[x(u)-z(u)]^{*} d u,[x(s)-z(s)]^{*}\right)+p(s)\right] d s \geq \frac{\lambda d R w}{2 r} \geq R
\end{aligned}
$$


which implies that $\|T x\| \geq\|x\|$ for all $x \in K \cap \partial \Omega_{R}$. It follows from Lemma 2.6 that

$$
i\left(T, K \cap \Omega_{R}, K\right)=0 .
$$

Combining (3.2) with (3.10) and the additivity of fixed point index, we know that

$$
i\left(T, K \cap\left(\bar{\Omega}_{R} \backslash \Omega_{r}\right), K\right)=i\left(T, K \cap \bar{\Omega}_{R}, K\right)-i\left(T, K \cap \Omega_{r}, K\right)=-1 .
$$

As a result, there exists $x^{*} \in K \cap\left(\bar{\Omega}_{R} \backslash \Omega_{r}\right)$ satisfying $T x^{*}=x^{*}$ and $r \leq\left\|x^{*}\right\| \leq R$. From (3.1) we have

$$
x^{*}(t)-z(t) \geq w\left\|x^{*}\right\|-\lambda G w \int_{0}^{1} p(s) d s \geq w\left\|x^{*}\right\|-r w>0 .
$$

Let $y(t)=x^{*}(t)-z(t)$. Then $y(t)$ is a positive solution of the problem (2.1). By virtue of Lemma 2.1. we know that $y(t)=\int_{0}^{t}(t-s) x^{*}(s) d s$ is a positive solution of the fourthorder multi-point boundary value problem (1.1). This completes the proof.

Remark 3.1. In the case, when $f=f(t, u)$ and $f$ has lower bound i. e. $f(t, u)+M \geq 0$ for some $M>0$, we can study the second-order multi-point boundary value problem under suitable condition by making use of the similar method. In particular, if $p(t)=$ $M$, the conclusion of Theorem 3.1. is still valid.

Remark 3.2. The constant $\lambda$ in problem (1.1) is usually called the Thiele modulus, in ap-plications, one is interested in showing the existence of positive solutions for semipositone problems for small enough $\lambda>0$.

\section{Examples}

Example 4.1. Consider the following singular fourth-order semipositone boundary value problem:

$$
\left\{\begin{array}{l}
y^{(4)}(t)+\frac{4 \lambda}{3 \sqrt[3]{(1-t)^{2}}}\left[\sin ^{8}\left(|y(t)|+\left|y^{\prime}(t)\right|\right)+e^{|y(t)|+\left|y^{\prime}(t)\right|+\left|y^{\prime \prime}(t)\right|}\right. \\
\left.+\left(|y(t)|+\left|y^{\prime}(t)\right|+\left|y^{\prime \prime}(t)\right|\right)^{\frac{1}{3}}\right]-\frac{2}{\sqrt{t}}=0, t \in(0,1) \\
y(0)=y^{\prime}(0)=0, \gamma^{\prime \prime}(1)=\frac{1}{3} y^{\prime \prime}\left(\frac{1}{2}\right), \quad y^{\prime \prime \prime}(0)=\frac{1}{4} y^{\prime \prime \prime}\left(\frac{1}{2}\right)
\end{array}\right.
$$

Proof. Let

$$
f\left(t, u_{1}, u_{2}, u_{3}\right)=\frac{4}{3 \sqrt[3]{(1-t)^{2}}}\left[\sin ^{8}\left(\left|u_{1}\right|+\left|u_{2}\right|\right)+e^{\left|u_{1}\right|+\left|u_{2}\right|+\left|u_{3}\right|}+\left(\left|u_{1}\right|+\left|u_{2}\right|+\left|u_{3}\right|\right)^{\frac{1}{3}}\right]-\frac{2}{\sqrt{t}}
$$

Then

$$
-p(t) \leq f\left(t, u_{1}, u_{2}, u_{3}\right) \leq q(t) g\left(u_{1}, u_{2}, u_{3}\right) \text { and } \lim _{\left(\left|u_{1}\right|+\left|u_{2}\right|+\left|u_{3}\right|\right) \rightarrow+\infty} \frac{f\left(t, u_{1}, u_{2}, u_{3}\right)}{\left|u_{1}\right|+\left|u_{2}\right|+\left|u_{3}\right|}=+\infty
$$

where

$$
p(t)=\frac{1}{\sqrt{t}}, q(t)=\frac{4}{3 \sqrt[3]{(1-t)^{2}}}
$$

$g\left(u_{1}, u_{2}, u_{3}\right)=\sin ^{8}\left(\left|u_{1}\right|+\left|u_{2}\right|\right)+e^{\left|u_{1}\right|+\left|u_{2}\right|+\left|u_{3}\right|}+\left(\left|u_{1}\right|+\left|u_{2}\right|+\left|u_{3}\right|\right)^{\frac{1}{3}}$, which implies that $\left(\mathbf{H}_{1}\right)-\left(\mathbf{H}_{3}\right)$ hold. Since $\alpha=\frac{1}{3}, \beta=\frac{1}{4}, \xi=\frac{1}{2}$, then we know that 


$$
\int_{0}^{1}(p(s)+q(s)) d s=\int_{0}^{1}\left(\frac{2}{\sqrt{s}}+\frac{4}{3 \sqrt[3]{(1-s)^{2}}}\right) d s=8 .
$$

Take $r=2$, then

$$
\begin{aligned}
& M_{1}=\max _{\left(\left|u_{1}\right|,\left|u_{2}\right|,\left|u_{3}\right|\right) \in[0,2] \times[0,2] \times[0,2]}\left\{g\left(\left|u_{1}\right|,\left|u_{2}\right|,\left|u_{3}\right|\right)\right\}+1 \\
& =\max _{\left(\left|u_{1}\right|,\left|u_{2}\right|,\left|u_{3}\right|\right) \in[0,2] \times[0,2] \times[0,2]}\left\{\left[\sin ^{8}\left(\left|u_{1}\right|+\left|u_{2}\right|\right)+e^{\left|u_{1}\right|+\left|u_{2}\right|+\left|u_{3}\right|}+\left(\left|u_{1}\right|+\left|u_{2}\right|+\left|u_{3}\right|\right)^{\frac{1}{3}}\right]\right\} \\
& +1=2+e^{6}+\sqrt[3]{6} . \\
& G=\left(\frac{1}{1-\sum_{i=1}^{m-2} \alpha_{i}}+\frac{\sum_{i=1}^{m-2} \beta_{i}\left(1-\sum_{i=1}^{m-2} \alpha_{i} \xi_{i}\right)}{\left(1-\sum_{i=1}^{m-2} \alpha_{i}\right)\left(1-\sum_{i=1}^{m-2} \beta_{i}\right)}\right) \frac{\left(1-\sum_{i=1}^{m-2} \alpha_{i} \xi_{i}\right)}{\sum_{i=1}^{m-2} \alpha_{i}\left(1-\xi_{i}\right)}=\frac{115}{12} . \\
& r\left[\frac{\frac{2}{m-s} d s}{G \int_{0}^{1} \frac{2}{\sqrt{s}} d s}=\frac{6}{115} .\right. \\
& {\left[\begin{array}{l}
M_{1} \int_{0}^{1}(p(s)+q(s)) d s\left(1-\sum_{i=1}^{m-2} \beta_{i} \sum_{i=1}^{m-2} \alpha_{i} \xi_{i}\right) \\
\left.\sum_{i=1}^{m-2} \alpha_{i}\right)\left(1-\sum_{i=1}^{m-2} \beta_{i}\right)
\end{array}\right]^{-1}=\frac{3}{23\left(2+e^{6}+\sqrt[3]{6}\right)} .}
\end{aligned}
$$

It follows from Theorem 3.1 that the problem (4.1) has at least one positive solution for any $\lambda^{*} \in\left(0, \frac{3}{23\left(2+e^{6}+\sqrt[3]{6}\right)}\right)$.

Example 4.2. Consider the following singular fourth-order semipositone boundary value problem:

$$
\left\{\begin{array}{l}
y^{(4)}(t)+\frac{8 \lambda}{\pi \sqrt{t(1-t)}}\left[\sin ^{19}\left(|y(t)|+\left|\gamma^{\prime}(t)\right|\right)+28 e^{|y(t)|+\left|y^{\prime}(t)\right|+\left|y^{\prime \prime}(t)\right|}+(|y(t)|\right. \\
\left.\left.+\left|y^{\prime}(t)\right|+\left|y^{\prime \prime}(t)\right|\right)^{\frac{1}{8}}\right]-\frac{3}{\sqrt{1-t}}=0, \quad t \in(0,1) \\
y(0)=y^{\prime}(0)=0, y^{\prime \prime}(1)=\frac{1}{3} y^{\prime \prime}\left(\frac{1}{2}\right)+\frac{1}{6} y^{\prime \prime}\left(\frac{2}{3}\right)+\frac{1}{10} y^{\prime \prime}\left(\frac{5}{6}\right) \\
y^{\prime \prime \prime}(0)=\frac{1}{6} y^{\prime \prime \prime}\left(\frac{1}{2}\right)+\frac{1}{4} y^{\prime \prime \prime}\left(\frac{2}{3}\right)+\frac{1}{10} y^{\prime \prime \prime}\left(\frac{5}{6}\right)
\end{array}\right.
$$

Proof. Let

$$
\begin{aligned}
f\left(t, u_{1}, u_{2}, u_{3}\right)= & \frac{8}{\pi \sqrt{t(1-t)}}\left[\sin ^{19}\left(\left|u_{1}\right|+\left|u_{2}\right|\right)+28 e^{\left|u_{1}\right|+\left|u_{2}\right|+\left|u_{3}\right|}+\left(\left|u_{1}\right|+\left|u_{2}\right|+\left|u_{3}\right|\right)^{\frac{1}{8}}\right] \\
& -\frac{3}{\sqrt{1-t}}
\end{aligned}
$$

Then

$$
-p(t) \leq f\left(t, u_{1}, u_{2}, u_{3}\right) \leq q(t) g\left(u_{1}, u_{2}, u_{3}\right) \text { and } \lim _{\left(\left|u_{1}\right|+\left|u_{2}\right|+\left|u_{3}\right|\right) \rightarrow+\infty} \frac{f\left(t, u_{1}, u_{2}, u_{3}\right)}{\left|u_{1}\right|+\left|u_{2}\right|+\left|u_{3}\right|}=+\infty
$$


where

$$
p(t)=\frac{3}{\sqrt{1-t}}, q(t)=\frac{8}{\pi \sqrt{t(1-t)}}
$$

$g\left(u_{1}, u_{2}, u_{3}\right)=\sin ^{19}\left(\left|u_{1}\right|+\left|u_{2}\right|\right)+28 e^{\left|u_{1}\right|+\left|u_{2}\right|+\left|u_{3}\right|}+\left(\left|u_{1}\right|+\left|u_{2}\right|+\left|u_{3}\right|\right)^{\frac{1}{8}}$, which implies that $\left(\mathbf{H}_{1}\right)-\left(\mathbf{H}_{3}\right) \quad$ hold. Since $\alpha_{1}=\frac{1}{3}, \alpha_{2}=\frac{1}{2}, \alpha_{3}=\frac{1}{5}, \xi_{1}=\frac{1}{2}, \xi_{2}=\frac{2}{3}, \xi_{3}=\frac{5}{6}, \beta_{1}=\frac{1}{6}, \beta_{2}=\frac{1}{4}, \beta_{3}=\frac{1}{10}$, then we know that

$$
\int_{0}^{1}(p(s)+q(s)) d s=\int_{0}^{1}\left(\frac{3}{\sqrt{1-s}}+\frac{8}{\pi \sqrt{s(1-s)}}\right) d s=6+8=14 .
$$

Take $r=3$, then

$$
\begin{aligned}
& M_{1}=\max _{\left(\left|u_{1}\right|,\left|u_{2}\right|,\left|u_{3}\right|\right) \in[0,3] \times[0,3] \times[0,3]}\left\{g\left(\left|u_{1}\right|,\left|u_{2}\right|,\left|u_{3}\right|\right)\right\}+1 \\
& =\max _{\left(\left|u_{1}\right|,\left|u_{2}\right|,\left|u_{3}\right|\right) \in[0,3] \times[0,3] \times[0,3]}\left\{\left[\sin ^{19}\left(\left|u_{1}\right|+\left|u_{2}\right|\right)+28 e^{\left|u_{1}\right|+\left|u_{2}\right|+\left|u_{3}\right|}+\left(\left|u_{1}\right|+\left|u_{2}\right|+\left|u_{3}\right|\right)^{\frac{1}{8}}\right]\right\} \\
& +1=2+28 e^{9}+\sqrt[4]{3} \text {. } \\
& G=\left(\frac{1}{1-\sum_{i=1}^{m-2} \alpha_{i}}+\frac{\sum_{i=1}^{m-2} \beta_{i}\left(1-\sum_{i=1}^{m-2} \alpha_{i} \xi_{i}\right)}{\left(1-\sum_{i=1}^{m-2} \alpha_{i}\right)\left(1-\sum_{i=1}^{m-2} \beta_{i}\right)}\right) \frac{\left(1-\sum_{i=1}^{m-2} \alpha_{i} \xi_{i}\right)}{\sum_{i=1}^{m-2} \alpha_{i}\left(1-\xi_{i}\right)} \\
& =\left(\frac{1}{\frac{2}{5}}+\frac{\frac{31}{60} \times \frac{23}{36}}{\frac{2}{5} \times \frac{29}{60}}\right) \times \frac{\frac{23}{36}}{\frac{43}{180}}=\frac{1010275}{89784} \text {. } \\
& \frac{r}{G \int_{0}^{1} p(s) d s}=\frac{3}{\frac{1010275}{89784} \times 6}=\frac{44892}{1010275} \text {. } \\
& r\left[\frac{M_{1} \int_{0}^{1}(p(s)+q(s)) d s\left(1-\sum_{i=1}^{m-2} \beta_{i} \sum_{i=1}^{m-2} \alpha_{i} \xi_{i}\right)}{\left(1-\sum_{i=1}^{m-2} \alpha_{i}\right)\left(1-\sum_{i=1}^{m-2} \beta_{i}\right)}\right]^{-1} \\
& =3 \times\left[\frac{\left(2+28 e^{9}+\sqrt[4]{3}\right) \times 14}{\frac{2}{5}} \times \frac{1-\frac{31}{60} \times \frac{13}{36}}{\frac{29}{60}}\right]^{-1}=\frac{3132}{61495\left(2+28 e^{9}+\sqrt[4]{3}\right)} \text {. }
\end{aligned}
$$

It follows from Theorem 3.1 that the problem (4.2) has at least one positive solution for any $\lambda^{*} \in\left(0, \frac{3132}{61495\left(2+28 e^{9}+\sqrt[4]{3}\right)}\right)$.

\section{Acknowledgements}

The author is very grateful to Editor of the Journal and the anonymous referees for their carefully reading of the first draft of the manuscript and making many valuable suggestions and comments. The author was supported financially by the Foundation of Shanghai Municipal Education Commission (Grant Nos. DZL803, 10YZ77, and DYL201105).

\section{Competing interests}

The author declares that she has no competing interests.

Received: 23 July 2011 Accepted: 9 February 2012 Published: 9 February 2012

\section{References}

1. Feng, W: On a m-point nonlinear boundary value problem. Nonlinear Anal. 30(6):5369-5374 (1997)

2. Feng, W, Webb, JRL: Solvability of a m-point boundary value problems with non-linear growth. J Math Anal Appl. 212, 467-480 (1997). doi:10.1006/jmaa.1997.5520

3. Gupta, CP: A generalized multi-point boundary value problem for second order or-dinary differential equation. Appl Math Comput. 89, 133-146 (1998). doi:10.1016/50096-3003(97)81653-0 
4. Gupta, CP: A Second order m-point boundary value problem at resonance. Nonlinear Anal. 24(10):1483-1489 (1995). doi:10.1016/0362-546X(94)00204-U

5. Gupta, CP, Ntouyas, SK, Tsamatos, P: On an m-point boundary value problem for second-order ordinary differential equations. Nonlinear Anal. 23, 1427-1436 (1994). doi:10.1016/0362-546X(94)90137-6

6. Gupta, CP, Ntouyas, SK, Tsamatos, P: Solvability of an $m$-point boundary value problem for second order ordinary differential equations. J Math Anal Appl. 189, 575-584 (1995). doi:10.1006/jmaa.1995.1036

7. Gupta, CP: Solvability of a three-point nonlinear boundary value problems for a second order ordinary differential equation. J Math Anal Appl. 168, 540-551 (1992). doi:10.1016/0022-247X(92)90179-H

8. Gupta, CP: A sharp condition for the solvability of a three-point second order boundary value problem. J Math Anal Appl. 205, 579-586 (1997)

9. Gupta, CP, Trofimchuk, S: Existence of a solution to a three-point boundary value problem and the spectral radius of a related linear operator. Nonlinear Anal. 34, 498-507 (1998)

10. Il'in, VA, Moiseev, El: Nonlocal boundary-value problem of the first kind for a Sturm-liouville operator in its differential and finite difference aspects. Diff Equ. 23, 803-810 (1987)

11. Krasnoselskii, MA: Positive Solutions of Operator Equation. Noordhoff, Groningen (1964)

12. Erbe, L, Wang, H: On the existence of positive solutions of ordinary differential equations. Proc Am Math Soc. 120 743-748 (1994). doi:10.1090/50002-9939-1994-1204373-9

13. Yao, Q: Existence of $n$ solutions and/or positive solutions to a semipositone elastic. Nonlinear Anal. 66, 138-150 (2007). doi:10.1016/j.na.2005.11.016

14. Yao, Q: Existence and multiplicity of positive solutions to a singular elastic beam equation rigidly fixed at both ends. Nonlinear Anal. 69, 2683-2694 (2008). doi:10.1016/j.na.2007.08.043

15. Yao, Q: Positive solutions of a nonlinear elastic beam equation rigidly fastened on the left and simply supported on the right. Nonlinear Anal. 69, 1570-1580 (2008). doi:10.1016/j.na.2007.07.002

16. Anuradha, V, Hai, DD, Shivaji, R: Existence results for superlinear semipositone BVPs. Proc Am Math Soc. 124, 757-746 (1996). doi:10.1090/50002-9939-96-03256-X

17. Guo, D, Lakshmikantham, V: Nonlinear Problems in Abstract Cone. Academic Press, New York (1988)

doi:10.1186/1687-2770-2012-12

Cite this article as: Sun: Existence of positive solutions for fourth-order semipositone multi-point boundary value problems with a sign-changing nonlinear term. Boundary Value Problems 2012 2012:12.

\section{Submit your manuscript to a SpringerOpen ${ }^{\odot}$} journal and benefit from:

- Convenient online submission

Rigorous peer review

- Immediate publication on acceptance

- Open access: articles freely available online

- High visibility within the field

- Retaining the copyright to your article

Submit your next manuscript at $\boldsymbol{s p r i n g e r o p e n . c o m ~}$ 